\title{
Compressibility of the high-pressure rocksalt phase of $\mathrm{ZnO}$
}

\author{
Recio, J.M.; Blanco, M.A.; Luana, V.; Pandey, R.; Gerward, Leif; Olsen, J. Staun
}

Published in:

Physical Review B

Link to article, DOI:

10.1103/PhysRevB.58.8949

Publication date:

1998

Document Version

Publisher's PDF, also known as Version of record

Link back to DTU Orbit

Citation (APA):

Recio, J. M., Blanco, M. A., Luana, V., Pandey, R., Gerward, L., \& Olsen, J. S. (1998). Compressibility of the high-pressure rocksalt phase of ZnO. Physical Review B, 58(14), 8949-8954.

https://doi.org/10.1103/PhysRevB.58.8949

\section{General rights}

Copyright and moral rights for the publications made accessible in the public portal are retained by the authors and/or other copyright owners and it is a condition of accessing publications that users recognise and abide by the legal requirements associated with these rights.

- Users may download and print one copy of any publication from the public portal for the purpose of private study or research.

- You may not further distribute the material or use it for any profit-making activity or commercial gain

- You may freely distribute the URL identifying the publication in the public portal

If you believe that this document breaches copyright please contact us providing details, and we will remove access to the work immediately and investigate your claim. 


\title{
Compressibility of the high-pressure rocksalt phase of $\mathrm{ZnO}$
}

\author{
J. M. Recio, M. A. Blanco, and V. Luaña \\ Departamento de Química Física y Analítica, Universidad de Oviedo, 33006-Oviedo, Spain \\ Ravindra Pandey \\ Department of Physics, Michigan Technological University, Houghton, Michigan 49931 \\ L. Gerward \\ Physics Department, Technical University of Denmark, Building 307, DK-2800 Lyngby, Denmark \\ J. Staun Olsen \\ Niels Bohr Institute, Oersted Laboratory, University of Copenhagen, DK-2100 Copenhagen, Denmark
}

(Received 13 March 1998)

\begin{abstract}
We report the results of a combined experimental and theoretical investigation on the stability and the volume behavior under hydrostatic pressure of the rocksalt $(B 1)$ phase of ZnO. Synchrotron-radiation x-ray powder-diffraction data are obtained from 0 to $30 \mathrm{GPa}$. Static simulations of the $\mathrm{ZnO} B 1$ phase are performed using the $a b$ initio perturbed ion method and the local and nonlocal approximations to the density-functional theory. After the pressure induced transition from the wurtzite phase, we have found that a large fraction of the $B 1$ high-pressure phase is retained when pressure is released. The metastability of this $\mathrm{ZnO}$ polymorph is confirmed through the theoretical evaluation of the Hessian eigenvalues of a nine-parameter potential energy surface. This allows us to treat the experimental and theoretical pressure-volume data on an equal basis. In both cases, we have obtained values of the bulk modulus in the range of 160-194 GPa. For its zero-pressure first derivative, the experimental and theoretical data yield a value of $4.4 \pm 1.0$. Overall, our results show that the $\mathrm{ZnO} B 1$ phase is slightly more compressible than previously reported. [S0163-1829(98)07537-7]
\end{abstract}

\section{INTRODUCTION}

There is a current interest in the derivation of the parameters that define the equation of state (EOS) of polymorphs that are unstable under room conditions. Basically, these parameters are three zero-pressure magnitudes: the volume $\left(V_{0}\right)$, the bulk modulus $\left(B_{0}\right)$, and its first derivative $\left(B_{0}^{\prime}\right)$. Attempts to extend standard empirical EOS, such as Birch ${ }^{1}$ and Vinet $^{2}$ analytical functions, to this situation have been discussed previously for the high-pressure phases of $\mathrm{KCl}$ (Ref. 3), ZnS (Refs. 4,5), and ZnO (Ref. 5). Very recently, Köhler et al. have shown that the first-order H11 form of Holzapfel $^{6}$ "perfectly represents" the EOS of the highpressure cesium chloride (B2) phase of the alkali halides. ${ }^{7}$ Besides, Hofmeister has also proposed in the last year a strategy to obtain $V_{0}, B_{0}$, and $B_{0}^{\prime}$ from infrared measurements on the $B 2$ phases of the alkali halides. ${ }^{8}$

The EOS of a pressure-induced polymorph can be unambiguously determined in the laboratory when the phase transition is irreversible enough to make the high-pressure phase metastable at ambient conditions. In other cases, theoretical calculations can be a useful alternative approach. For instance, theoretical analysis can be used to select the best set of structural parameters among a series of extrapolated values to zero pressure from high-pressure data. ${ }^{5}$ Also, a theoretical scheme can give, after calibration to high-pressure polymorphs, reliable EOS parameters, as documented in the case of the orthorhombic phase of $\mathrm{CaF}_{2}$, where the results of $a b$ initio perturbed ion model (ai $\mathrm{PI})$ were compared with synchrotron-radiation x-ray-diffraction data. ${ }^{9}$

An important example where this structural characterization needs further investigation is $\mathrm{ZnO}$. This crystal under- goes a pressure-induced phase transition from the wurtzite $(B 4)$ to the rocksalt $(B 1)$ phase in the range of $10 \mathrm{GPa}$, as reported by Bates, White, and Roy. ${ }^{10}$ These authors found that the $B 1$ phase can be quenched, since no trend to revert to the $B 4$ form was found even above $100{ }^{\circ} \mathrm{C}$. However, using in situ $\mathrm{x}$-ray diffraction, Jamieson ${ }^{11}$ found this transition to be reversible at room temperature. More recently, Karzel et al. reported a large hysteresis for this transition from $\mathrm{x}$-ray and ${ }^{67} \mathrm{Zn}$-Mössbauer spectroscopy studies, although they did not give data for the $B 1$ phase below 2.0 $\pm 0.5 \mathrm{GPa} .{ }^{12}$ In a preliminary study ${ }^{13}$ we have found that a large fraction of the $B 1$ phase is retained at room temperature and ambient pressure.

The few quantum-mechanical studies directed to calculate $V_{0}, B_{0}$, and $B_{0}^{\prime}$ of the $\mathrm{ZnO} B 1$ phase leave a number of questions unsolved. Our previous aiPI calculations ${ }^{5}$ gave a value for $B_{0}$ rather different from that reported by Jaffe and Hess ${ }^{14}$ using the CRYSTAL approach. ${ }^{15}$ These two HartreeFock calculations did not include electron correlation, a correction that can increase the bulk modulus by more than $20 \% .{ }^{16}$ In addition, Karzel et al. complemented their experimental work with linearized-augmented plane-wave calculations in the $\mathrm{ZnO} B 1$ phase. ${ }^{12}$ We note, however, that they did not provide a full determination of the $\mathrm{ZnO}$ EOS since the value of $V_{0}$ was not reported.

In this paper, we pursue a twofold objective which addresses the question of the stability of the $B 1$ phase of $\mathrm{ZnO}$ at zero pressure conditions along with the accurate determination of its EOS parameters. To undertake such investigation, we have combined experimental measurements with quantum-mechanical computations. The experimental data are collected through synchrotron-radiation x-ray diffraction 


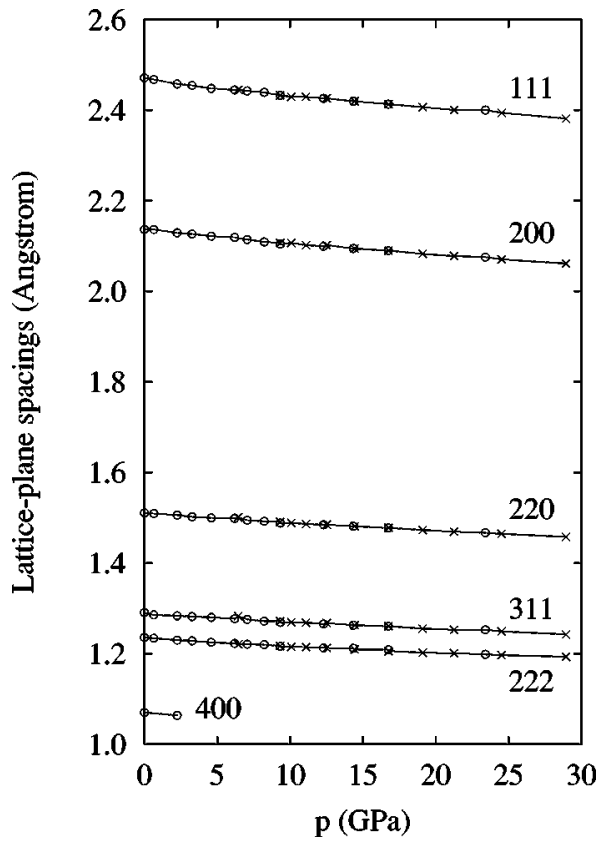

FIG. 1. Lattice-plane spacings as functions of pressure for the $\mathrm{ZnO} B 1$ phase. Crosses denote increasing pressure and circles decreasing pressure. Miller indices $h k l$ are given.

at pressures obtained in a diamond-anvil cell. The computations are performed using the aiPI methodology including correlation energy corrections, ${ }^{17,18}$ and the local and nonlocal density-functional theory (DFT) approximations as implemented in the CRYSTAL program. ${ }^{15}$ Our experimental observations confirm that the $B 4-B 1$ transition takes place at 10 $\mathrm{GPa}$ and the high-pressure phase appears to be metastable at ambient conditions. Our calculations reveal the existence of a local minimum at the $B 1$ configuration in a nine-parameter energy surface. From our observed data and quantummechanical calculations, we report the best values of $V_{0}, B_{0}$, and $B_{0}^{\prime}$ for the EOS of the $B 1$ phase of $\mathrm{ZnO}$.

The experimental procedure and the computational details are presented in the next section. Section III contains the results and the discussion on the metastability and the EOS. Our concluding remarks are summarized in the last section.

\section{EXPERIMENTAL PROCEDURE AND COMPUTATIONAL TECHNIQUES}

\section{A. Pressure-volume measurements}

$\mathrm{X}$-ray powder-diffraction spectra were recorded using synchrotron radiation and the white-beam, energy-dispersive method. High pressures were obtained in a SyassenHolzapfel type ${ }^{19}$ diamond-anvil cell. The sample and a small ruby chip were enclosed in a hole of diameter $0.1 \mathrm{~mm}$ in an inconel gasket. The incident-beam cross section was 60 $\times 60 \mu \mathrm{m}^{2}$. A 4:1 methanol:ethanol mixture was used as the pressure-transmitting medium, and the pressure was determined from the wavelength shift of the ruby $R$ line, applying the nonlinear pressure scale of Mao et al. ${ }^{20}$ The uncertainty in the pressure determination is estimated to be $0.1 \mathrm{GPa}$ for pressures below about $10 \mathrm{GPa}$. For higher pressures the uncertainty may be larger because of nonhydrostatic conditions as will be discussed below. The diffraction data were ob-

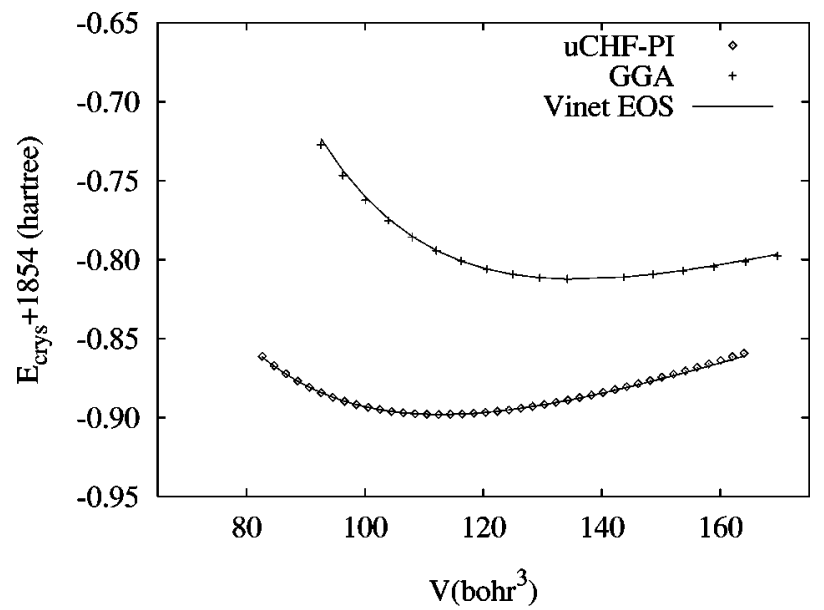

FIG. 2. Crystal energy of the $\mathrm{ZnO} B 1$ phase according to uCHF-PI and GGA calculations. The corresponding integrated Vinet EOS fittings are also shown.

tained at the UK synchrotron radiation source of the Daresbury Laboratory, and at the synchrotron radiation laboratory HASYLAB in Hamburg, Germany. All experiments were performed at room temperature. The diffraction peaks in the recorded spectra yield information on the corresponding plane spacings, and the unit-cell volume is deduced from the refined lattice constants.

\section{B. Total energy calculations}

We have followed two different theoretical approaches to compute the total energy of the $\mathrm{ZnO} B 1$ phase. The first one is the aiPI model, a quantum-mechanical method that solves the Hartree-Fock (HF) equations of the solid in a localized Fock space by breaking the crystal wave function into local, group functions (atomic or ionic in nature). ${ }^{21}$ For a detailed description of the computational implementation and its latest update, we submit to Refs. 17 and 18. In the present work, we have used for $\mathrm{Zn}^{2+}$ and $\mathrm{O}^{2-}$ the nearly HF multi$\zeta$ exponential Clementi and Roetti basis sets ${ }^{22}$ of $\mathrm{Zn}^{2+}$ and $\mathrm{O}^{-}$. After each ai PI calculation, a correlation energy correction is added to the total energy of the crystal using the Coulomb-Hartree-Fock method of Clementi. ${ }^{23}$ These calculations are denoted as UCHF-PI.

As a second theoretical tool, we have used the implementation of the DFT methodology in a periodic linearcombination-of-atomic-orbitals (LCAO) scheme included in the crystal program. ${ }^{15}$ In the LCAO approach, the Bloch functions are constructed as linear combinations of atomcentered Gaussian orbitals. We have considered the PerdewZunger parametrization ${ }^{24}$ of Ceperley-Alder results ${ }^{25}$ for the local functional calculations (referred to as LDA), and the combination of the Becke exchange functional ${ }^{26}$ with the Perdew-Wang correlation functional ${ }^{27}$ for the nonlocal case (referred to as GGA). In this work, we have selected the same basis sets for $\mathrm{Zn}$ and $\mathrm{O}$ that Jaffe and Hess used in their $\mathrm{HF}$ calculations of $\mathrm{ZnO}$ polymorphs. ${ }^{14}$ This fact allows a direct comparison of their results with the local and nonlocal computations performed here. 
TABLE I. Summary of EOS parameters for the $B 1$ phase of $\mathrm{ZnO}$.

\begin{tabular}{lcccc}
\hline \hline & Method & $a_{0}(\AA)$ & $B_{0}(\mathrm{GPa})$ & $B_{0}^{\prime}$ \\
\hline Ref. 10 & X-ray & 4.280 & & \\
Ref. 12 & X-ray & 4.271 & 228 & 4 (fix) \\
Ref. 5 & HF-PI & 4.225 & $132^{\mathrm{b}}$ & $3.8^{\mathrm{b}}$ \\
Ref. 14 & HF-CRYSTAL & 4.294 & 203.3 & 3.6 \\
Ref. 12 & LAPW & & 205 & 4.88 \\
This work & X-ray & 4.275 & $194^{\mathrm{a}}-194^{\mathrm{b}}$ & $4.8^{\mathrm{a}}-4.7^{\mathrm{b}}$ \\
This work & uCHF-PI & 4.058 & $170^{\mathrm{a}}-160^{\mathrm{b}}-169^{\mathrm{c}}$ & $3.37^{\mathrm{a}}-4.97^{\mathrm{b}}-3.55^{\mathrm{c}}$ \\
This work & GGA & 4.316 & $176^{\mathrm{a}}-180^{\mathrm{b}}-175^{\mathrm{c}}$ & $5.39^{\mathrm{a}}-4.69^{\mathrm{b}}-5.35^{\mathrm{c}}$ \\
This work & LDA & 4.207 & $229^{\mathrm{a}}-226^{\mathrm{b}}-229^{\mathrm{c}}$ & $4.24^{\mathrm{a}}-4.62^{\mathrm{b}}-4.24^{\mathrm{c}}$ \\
\hline \hline
\end{tabular}

${ }^{\mathrm{a} B i r c h}$ EOS fitting.

${ }^{\mathrm{b}} \mathrm{H} 11$ EOS fitting.

${ }^{c}$ Vinet EOS fitting.

\section{RESULTS AND DISCUSSION}

\section{A. Thermodynamic stability of the $\mathrm{ZnO}$ rocksalt phase}

In our $\mathrm{x}$-ray-diffraction experiments, it is observed that the hexagonal wurtzite structure of $\mathrm{ZnO}$ undergoes a structural phase transformation with a transition pressure $p_{\text {tr }}$ $\simeq 10 \mathrm{GPa}$. It is around this pressure where the diffraction lines of the high-pressure rocksalt phase begin to appear. The transition is completed at about $15 \mathrm{GPa}$. The transition pressure is in good agreement with the experimental value 9.5 GPa tabulated by Phillips, ${ }^{28}$ and slightly greater than the recent value $8.7 \pm 0.5 \mathrm{GPa}$ obtained also in x-ray-diffraction experiments by Karzel et al. ${ }^{12}$ With respect to our theoretical predictions, we have obtained different values of the thermodynamic $p_{\text {tr }}$ depending on the computational method. It is known that under the consideration of spherical electronic densities, as in the aiPI model, the $B 1$ phase of $\mathrm{ZnO}$ is the structure with lower energy at zero pressure (see Ref. 29). Incorporation of nonspherical deformations through GGA calculations yield a value for the thermodynamic $p_{\text {tr }}$ around $9 \mathrm{GPa}$, close to the experimental range. As expected, the LDA prediction is lower by about $5 \mathrm{GPa}^{16}$

The measured lattice-plane spacings as functions of pressure for the $B 1$ phase are shown in Fig. 1. Accordingly, a large fraction of the $B 1$ phase is retained when the pressure is released. In this way, the $B 1$ phase of $\mathrm{ZnO}$ is found to be metastable at zero pressure, and $V_{0}$ for this structure can be determined. The lattice parameter is $a_{0}=4.275 \pm 0.004 \AA$ in good agreement with the value $4.280 \AA$ reported by Bates et al. ${ }^{10}$ and the value $4.271 \pm 0.002 \AA$ given by Karzel et al. ${ }^{12}$ However, in this reference the ambient pressure data are extrapolated from higher pressures, since below $\simeq 2 \mathrm{GPa}$ their $B 1$ phase was completely transformed back to the wurtzite structure.

In the theoretical study of the $B 1$ phase, we have first computed the total energy of this structure at volumes ranging approximately from 0.6 to 1.2 times the experimental equilibrium value. ${ }^{10}$ The results for the uCHF-PI and GGA calculations are plotted in Fig. 2. The calculated equilibrium volumes yield the static lattice parameter of $4.058 \AA$ at the uCHF-PI and $4.316 \AA$ at the GGA. If we compare the present uCHF-PI with the HF-PI calculation previously published, ${ }^{5}$ we see that the stabilization effect of the correlation energy correction is greater at lower volumes leading to a decreasing of the lattice parameter with respect to the $\mathrm{HF}$ (PI) result, as expected. The uCHF-PI lattice parameter is now 5\% smaller than the experimental value. The use of nonlocal functionals, on the other hand, produces also the expected upwards shift in the equilibrium lattice spacing (see Table I). ${ }^{16}$ The GGA equilibrium geometry is about $1 \%$ larger than the experimental value.

Once the equilibrium volume is located, we explore the response of the $B 1$ structure to general strains in two different ways. First, we seek for small perturbations from the equilibrium restricted to a primitive rhombohedral unit cell containing one $\mathrm{ZnO}$ molecule: the cation is at the origin of the cell, the anion at $(x, x, x)$, and the space group is $R 3 m$. Therefore, we allow the crystal to search lower energy situations in a three-dimensional (3D) configuration space, where the three degrees of freedom are the unit-cell length and angle ( $a$ and $\alpha$ ), and the internal coordinate $x$. In this $3 \mathrm{D}$ model, the $B 1$ phase corresponds to the point for which

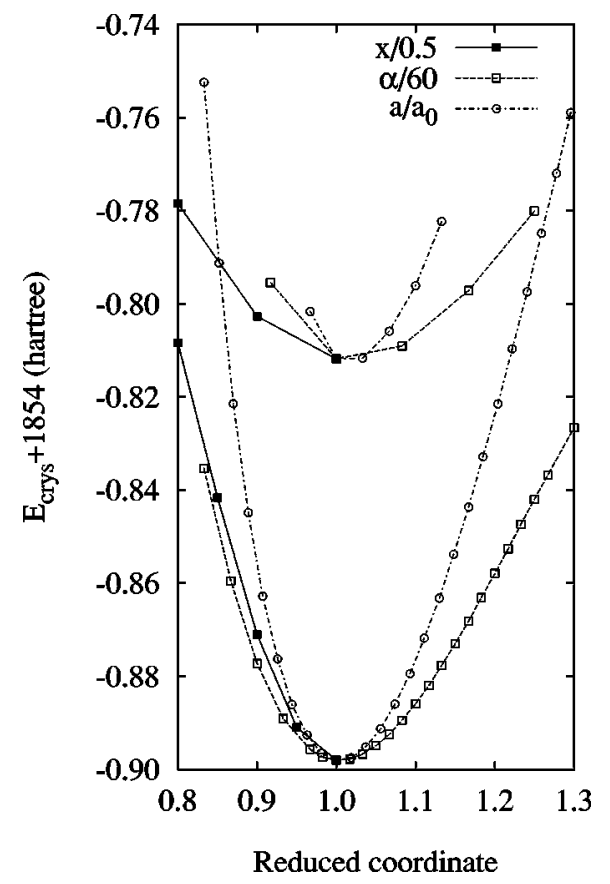

FIG. 3. Crystal energy of $\mathrm{ZnO}$ as a function of $R 3 \mathrm{~m}$ unit-cell reduced parameters according to uCHF-PI (below) and GGA (above) calculations. 
$x=1 / 2, \quad \alpha=60^{\circ}$, and $a=a_{0} / \sqrt{2}$, being $a_{0}$ the equilibrium lattice parameter previously computed.

In Fig. 3, we plot the energy curves for the displacement of these three parameters from their $B 1$ values. It is found that all departures from the equilibrium are accompanied by a rising in the crystal energy, and so the $B 1$ phase is a stable minimum with respect to these three deformations independently. It is worth noting that GGA calculations predict softer curves for $\alpha$ and $x$ displacements than uCHF-PI. This effect is related to the neglect of multipolar terms in aiPI, which are stabilizing contributions for the lower symmetry situations out of the minimum. Nevertheless, both calculations predict that the softer curves are those arising from the variation of $\alpha$. We are currently working in the description of a possible transition mechanism to the $B 1$ structure from the $B 3$ (zinc-blende) phase. This form can be described within the same $3 \mathrm{D}$ space $\left(x=1 / 4, \alpha=60^{\circ}\right)$, and its structure closely resembles that of the ambient pressure polymorph, the $B 4$ (wurtzite) phase.

We have also completed a more general and detailed investigation of the stability of the $B 1$ phase by relaxing the symmetry constrains of the rhombohedral cell. We have considered the case of $a \neq b \neq c, \alpha \neq \beta \neq \gamma$ and $x \neq y \neq z$. This nine parameter (9D) model is analogous to the one used in our previous study of the microscopic description of the $B 1-B 2$ phase transition. ${ }^{30}$ In order to prove that the $B 1$ phase of $\mathrm{ZnO}$ is stable, and thus a minimum in this 9D space, we determine and diagonalize its $9 \times 9$ Hessian matrix at the equilibrium volume. Given the high symmetry of the $B 1$ phase, this matrix shows a blocked structure,

$$
\mathbf{H}^{B 1}=\left(\begin{array}{ccccccccc}
H_{a a} & H_{a b} & H_{a b} & H_{a \alpha} & H_{a \beta} & H_{a \beta} & 0 & 0 & 0 \\
H_{a b} & H_{a a} & H_{a b} & H_{a \beta} & H_{a \alpha} & H_{a \beta} & 0 & 0 & 0 \\
H_{a b} & H_{a b} & H_{a a} & H_{a \beta} & H_{a \beta} & H_{a \alpha} & 0 & 0 & 0 \\
H_{a \alpha} & H_{a \beta} & H_{a \beta} & H_{\alpha \alpha} & H_{\alpha \beta} & H_{\alpha \beta} & 0 & 0 & 0 \\
H_{a \beta} & H_{a \alpha} & H_{a \beta} & H_{\alpha \beta} & H_{\alpha \alpha} & H_{\alpha \beta} & 0 & 0 & 0 \\
H_{a \beta} & H_{a \beta} & H_{a \alpha} & H_{\alpha \beta} & H_{\alpha \beta} & H_{\alpha \alpha} & 0 & 0 & 0 \\
0 & 0 & 0 & 0 & 0 & 0 & H_{x x} & H_{x y} & H_{x y} \\
0 & 0 & 0 & 0 & 0 & 0 & H_{x y} & H_{x x} & H_{x y} \\
0 & 0 & 0 & 0 & 0 & 0 & H_{x y} & H_{x y} & H_{x x}
\end{array}\right) .
$$

The eigenvalues and eigenvectors of this matrix can be calculated analytically. However, the numerical evaluation of eight different second derivatives of the crystal energy is still a formidable task, and so we use the computationally less expensive uCHF-PI method to obtain the Hessian matrix elements. Our main finding is that all the eigenvalues are positive and, therefore, the $B 1$ phase lies in a true local minimum of the 9D model according to our calculations. This result is consistent with the metastability of this phase observed in the $\mathrm{x}$-ray experiments.

\section{B. Equation of state of the $\mathrm{ZnO}$ rocksalt phase}

The pressure dependence of the volume per formula unit can be calculated from the refined unit-cell parameters using the experimental data plotted in Fig. 1. The set of pressurevolume $(p-V)$ points obtained in this way has subsequently been introduced as input for Birch, Vinet, and H11 fittings. It is to be noted that the number of free parameters in the three functions is, respectively, three, two, and one. The EOS parameters from these fittings are tabulated in Table I. Values for the pressure derivative $B_{0}^{\prime}$ are typically between 4 and 5 for most solids. It is seen in Table I that all tabulated $B_{0}^{\prime}$ values, experimental as well as theoretical, fall approximately within this range. In the present work, we have fitted the EOS to the experimental data points for pressures below $11 \mathrm{GPa}$, and the resulting $B_{0}$ and $B_{0}^{\prime}$ values are given in
Table I. Around $11 \mathrm{GPa}$, the methanol:ethanol mixture is known to freeze, and nonhydrostatic conditions may be introduced in the diamond-anvil cell. ${ }^{31}$ Fitting the EOS to all our experimental data in the whole observed pressure range $p<30 \mathrm{GPa}$ results in $B_{0}^{\prime}=6.6$, i.e., a value that is larger than expected. We believe that the freezing of the pressure transmitting medium cause the observed slope change in our experimental pressure-volume data. The previous experimental $B_{0}$ and $B_{0}^{\prime}$ values yield a description of a less compressible $\mathrm{ZnO} B 1$ phase. Thus, the $\mathrm{x}$-ray data by Karzel et al. ${ }^{12}$ give a $B_{0}$ value which is about $35 \mathrm{GPa}$ above our experimental value. Their slightly lower value for $B_{0}^{\prime}$ can only explain partially this large disagreement. We should notice two facts here. First, Karzel et al. EOS parameters come from an extrapolation to zero pressure of data in the pressure range 2-10 GPa. Second, their $B_{0}$ value for the $\mathrm{ZnO} B 4$ phase is also $\simeq 40 \mathrm{GPa}$ greater than previously reported. 32,33

We have also generated $p-V$ tables from the computed crystal energy $\left(E_{\text {crys }}\right)$ values by minimizing the static Gibbs energy $\left(G=E_{\text {crys }}+p V\right)$ with respect to $V$ at selected values of the hydrostatic pressure in the range 0-30 GPa. The $p-V$ tables were then used to generate $B_{0}$ and $B_{0}^{\prime}$ by means, again, of Birch, Vinet, and H11 fittings. The values are included in Table I. We have obtained in the three cases very good fittings to both uCHF-PI and GGA data. The quality of 


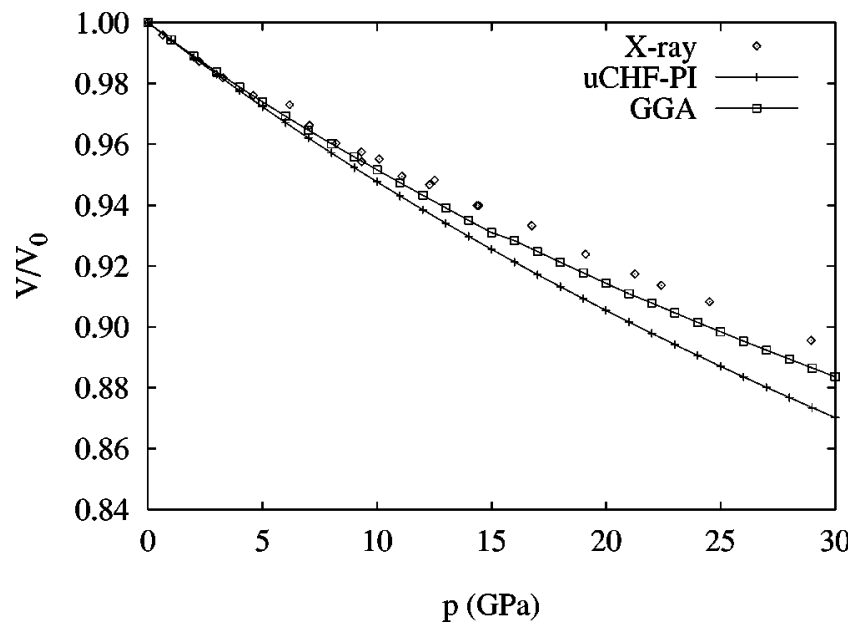

FIG. 4. $V / V_{0}-p$ diagram according to our $\mathrm{x}$-ray-diffraction data and uCHF-PI and GGA calculations for the $\mathrm{ZnO} B 1$ phase.

the fittings is illustrated in Fig. 2, where we plot the computed uCHF-PI and GGA $E_{\text {crys }}$ values along with the curves obtained after the integration of the corresponding Vinet EOS.

To represent the data from our experiments and computations, we have adopted the usual $V / V_{0}$ versus $p$ diagram, $V_{0}$ being the zero-pressure volume of the $B 1$ phase (see Fig. 4). In this normalized representation, both uCHF-PI and GGA curves show very good agreement with the experimental data in the pressure region below the transition pressure $(0-10$ $\mathrm{GPa}$ ). Above this value, it is found a slow progressive departure of the theory with respect to the experiment, reaching a $2 \%$ difference at the highest pressure measured (30 GPa). Due to this fact, the $\mathrm{ZnO} B 1$ phase is described as more compressible in our computations. This picture is consistent with the values obtained for $B_{0}$ and $B_{0}^{\prime}$. In our work, experimental and theoretical $B_{0}$ values are in the range of 160-194 $\mathrm{GPa}$, and the slope change in the experimental data is due to the freezing of the pressure transmitting medium as discussed above.

The comparison with other calculations ${ }^{12,14}$ shows a better agreement than with the experiments of Karzel et al. ${ }^{12}$ although in general our computations yield a slightly more compressible $\mathrm{ZnO} \quad B 1$ phase than previously predicted. The difference between $B_{0}$ values computed with and without correlation effects shows an opposite trend to the difference in the prediction of $a_{0}$, as mentioned previously. ${ }^{9,16}$ The same can be said for the results under DFT when comparing local and nonlocal functionals. ${ }^{16}$ We notice that the improvements in the theoretical approaches (i.e., inclusion of correlation energy corrections and use of nonlocal density functionals) change the predictions of $a_{0}$ and $B_{0}$ in a different direction. Thus, the uCHF-PI $B_{0}$ value is about $40 \mathrm{GPa}$ higher than the HF-PI one, and the corresponding GGA value is lower than the LDA one by approximately $50 \mathrm{GPa}$. Finally, it is to be stressed that given the different formalisms upon which the two quantum-mechanical methodologies are based, the computed EOS parameters show an excellent agreement with each other and globally describe satisfactorily our $\mathrm{x}$-ray-diffraction data.

\section{CONCLUDING REMARKS}

In this paper, we have reported the results of experiments and calculations directed (i) to investigate the thermodynamic stability of the high-pressure $B 1$ phase of $\mathrm{ZnO}$, and (ii) to determine the EOS parameters of this polymorph. The synchrotron-radiation $x$-ray diffraction informs us of a pressure-induced transition from the $B 4$ to the $B 1$ phase around $10 \mathrm{GPa}$. The $B 1$ phase is retained metastable at ambient conditions. Our quantum-mechanical calculations have confirmed the existence of a local minimum located at the $B 1$ position on a nine-dimensional energy surface.

The experimental and calculated results have been used to obtain the $p-V$ behavior of the $\mathrm{ZnO} B 1$ phase. $B_{0}$ and $B_{0}^{\prime}$ values are generated using the standard Birch and Vinet empirical EOS along with the semiempirical H11 EOS. The most interesting conclusions are: (i) our four different sets of data (one experimental and three theoretical) can be consistently represented by these EOS giving in each case very similar Birch, Vinet, and H11 EOS parameters; (ii) $B_{0}$ is obtained in the range 160-194 GPa in the experiments and in the theoretically improved uCHF-PI and GGA calculations; (iii) freezing of the pressure transmitting medium causes a change in slope of the experimental pressurevolume curve around $11 \mathrm{GPa}$ that explains the differences with respect to the computed results; and (iv) the global comparison with previous experiments and other calculations shows that the $\mathrm{ZnO} B 1$ phase is slightly more compressible than reported before.

\section{ACKNOWLEDGMENTS}

We thank Daresbury Laboratory for use of the SRS under the EU Large-Scale Facilities Program, and HASYLABDESY for permission to use the synchrotron radiation facility. Financial support from the Danish Natural Science Research Council and Spanish DGICYT, Project No. PB960559 , are gratefully acknowledged. J.M.R. is grateful to the Ministerio de Educacion y Cultura (DGES) of Spain and the Department of Physics of MTU for financial support during his stay at Michigan Technological University.
${ }^{1}$ F. Birch, Phys. Rev. 71, 809 (1947).

${ }^{2}$ P. Vinet, J. Ferrante, J. H. Rose, and J. R. Smith, J. Geophys. Res. 92, 9319 (1987).

${ }^{3}$ A. J. Campbell and D. L. Heinz, J. Phys. Chem. Solids 52, 495 (1991).
${ }^{4}$ Y. Zhuo, A. J. Campbell, and D. L. Heinz, J. Phys. Chem. Solids 52, 821 (1991).

5 J. M. Recio, R. Pandey, and V. Luaña, Phys. Rev. B 47, 3401 (1993).

${ }^{6}$ W. B. Holzapfel, Europhys. Lett. 16, 67 (1991). 
${ }^{7}$ U. Köhler, P. G. Johansen, and W. P. Holzapfel, J. Phys.: Condens. Matter 9, 5581 (1997).

${ }^{8}$ A. M. Hofmeister, Phys. Rev. B 56, 5835 (1997).

${ }^{9}$ A. Martín Pendás, J. M. Recio, M. Flórez, V. Luaña, and M. Bermejo, Phys. Rev. B 49, 5858 (1994).

${ }^{10}$ C. H. Bates, W. B. White, and R. Roy, Science 137, 993 (1962).

${ }^{11}$ J. C. Jamieson, Phys. Earth Planet. Inter. 3, 201 (1970).

${ }^{12}$ H. Karzel, W. Potzel, M. Kofferlein, W. Schiessl, M. Steiner, U. Hiller, G. M. Kalvius, D. W. Mitchell, T. P. Das, P. Blaha, K. Schwarz, and M. P. Pasternak, Phys. Rev. B 53, 11425 (1996).

${ }^{13}$ L. Gerward and J. S. Olsen, J. Synchrotron Radiat. 2, 233 (1995).

${ }^{14}$ J. E. Jaffe and A. C. Hess, Phys. Rev. B 48, 7903 (1993).

${ }^{15}$ R. Dovesi, V. R. Saunders, C. Roetti, M. Causà, N. M. Harrison, R. Orlando, and E. Aprà, CRYSTAL95 User's Manual, Universidad de Torino, 1996.

${ }^{16}$ R. Pandey, M. Causà, N. M. Harrison, and M. Seel, J. Phys.: Condens. Matter 8, 3993 (1996).

${ }^{17}$ V. Luaña, A. Martín Pendás, J. M. Recio, E. Francisco, and M. Bermejo, Comput. Phys. Commun. 77, 107 (1993).

${ }^{18}$ M. A. Blanco, V. Luã̃a and A. Martín Pendás, Comput. Phys. Commun. 103, 287 (1997).

${ }^{19}$ G. Huber, K. Syassen, and W. B. Holzapfel, Phys. Rev. B 15,
5123 (1977).

${ }^{20}$ H. K. Mao, P. M. Bell, J. W. Shaner, and D. J. Steinberg, J. Appl. Phys. 49, 3276 (1978).

${ }^{21}$ V. Luaña, and L. Pueyo, Phys. Rev. B 41, 3800 (1990).

${ }^{22}$ E. Clementi and C. Roetti, At. Data Nucl. Data Tables 14, 177 (1974).

${ }^{23}$ J. Chakravorty and E. Clementi, Phys. Rev. A 39, 2290 (1989), and references therein.

${ }^{24}$ J. P. Perdew and A. Zunger, Phys. Rev. B 23, 5048 (1981).

${ }^{25}$ D. M. Ceperley and B. J. Alder, Phys. Rev. Lett. 45, 566 (1980).

${ }^{26}$ A. D. Becke, Phys. Rev. A 38, 3098 (1988).

${ }^{27}$ J. P. Perdew, Electronic Structure of Solids '91, edited by P. Ziesche and H. Eschrig (Academic, Berlin, 1991).

${ }^{28}$ J. C. Phillips, Phys. Rev. Lett. 27, 1197 (1971).

${ }^{29}$ M. Wilson and P. A. Madden, Mol. Phys. 97, 75 (1997).

${ }^{30}$ A. Martín Pendás, V. Luaña, J. M. Recio, M. Flórez, E. Francisco, M. A. Blanco, and L. N. Kantorovich, Phys. Rev. B 49, 3066 (1994).

${ }^{31}$ R. T. Downs, C. S. Zha, T. S. Duffy, and L. W. Finger, Am. Mineral. 81, 51 (1996).

${ }^{32}$ T. B. Bateman, J. Appl. Phys. 33, 3309 (1962).

${ }^{33}$ I. B. Kobiakov, Solid State Commun. 35, 305 (1980). 